 \\ ISSN: 2448-0215 (VERSÃO ON-LINE)
}

\section{UNA METÁFORA PARA DESCRIBIR \\ EL APRENDIZAJE CON BASE EN LA FÍSICA CUÁNTICA}

\author{
José Ángel García Retana \\ Universidad de Costa Rica \\ jose.garcia@ucr.ac.cr \\ Greivin Hernández González \\ Universidad de Costa Rica \\ greivin.hernandez@ucr.ac.cr
}

\section{Resumen:}

El aprendizaje es un proceso complejo, en el que intervienen y se vinculan múltiples variables. La construcción del aprendizaje es un acto individual que se desarrolla en un contexto natural y social. Ninguno de estos aspectos se puede considerar como nuevo, sin embargo el visualizar el aprendizaje y todas esas características tomando en cuenta la dinámica de las partículas propuesta desde la perspectiva de la Física cuántica, nos ofrece un nuevo modelo para reflexionar sobre este aspecto.

Palabras claves: Aprendizaje, Física cuántica, metáfora

\begin{abstract}
:
El aprendizaje es un proceso complejo, en el que intervienen y se vinculan múltiples variables. La construcción del aprendizaje es un acto individual que se desarrolla en un contexto natural y social. Ninguno de estos aspectos se puede considerar como nuevo, sin embargo el visualizar el aprendizaje y todas esas características tomando en cuenta la dinámica de las partículas propuesta desde la perspectiva de la Física cuántica, nos ofrece un nuevo modelo para reflexionar sobre este aspecto.
\end{abstract}

Key words: Aprendizaje, Física cuántica, metáfora 


\section{Introducción}

El modelo educativo, como institución social que procura la transformación de los individuos, debe ir más allá de abastecer a las comunidades de maestros e instalaciones (Capra, 1992), y centrarse en el problema del aprendizaje, por cuanto es este el factor que genera tal transformación. En el presente ensayo se considera al aprendizaje como un proceso complejo que involucra un gran número de factores; el mismo es un acto individual a través del cual el aprendiz logra transformar la información que recibe desde su entorno (natural, social e interno), en conocimiento o habilidad, en calidad de inserción introspectiva en sus estructuras mentales con un carácter significativo (García, 2011).

Al respecto, Cotton (1989), citado por Cantú (2003), indica que el aprendizaje consiste en un proceso de adquisición de nuevos conocimientos o habilidades y que, para que los mismos puedan ser calificados como como tales, deben ir más allá de una simple retención pasajera, y poder manifestarse posteriormente. Lo anterior implica que el aprendizaje conlleva la integración de nuevos elementos en las estructuras mentales que el individuo posee pero que no existían o no estaban ligadas entre sí, formando una nueva estructura que permite procesar nueva información (De Natale, 1990, citado por Saldaña, 2010), por ello el aprendizaje es dinámico y creciente.

En las sociedades occidentales, muchos de los modelos educativos han partido de la concepción de la enseñanza-aprendizaje en calidad de causa/efecto, enmarcándose dentro de la concepción cartesiana-newtoniana que ha prevalecido en los últimos trecientos años. En el presente ensayo se rompe con esa dirección y se persigue valorar el proceso de aprendizaje de manera independiente al de la enseñanza; desde la perspectiva del pensamiento complejo. Se considerará que el aprendizaje deviene en el resultado de la interacción de múltiples factores, entre los que figuran: la cognición, la emoción, la comunicabilidad, las características fisiológicas, los estilos de aprendizaje, el espacio/tiempo, aspectos que se vinculan e interactúan simultáneamente, por lo cual no responde al pensamiento simplificado de causa-efecto. Estos factores no son los únicos que definen el proceso de aprendizaje, pero serán los que se tomarán en cuenta porque los mismos contribuyen a formular una metáfora que describe la construcción del aprendizaje a partir de los constructos fundamentales de la física cuántica. 


\section{Una metáfora para describir el aprendizaje a partir de la Física Cuántica}

En los años ochenta del siglo pasado cuando Howard Gardner propuso su teoría sobre las inteligencias múltiples, advirtió que el aprendizaje está ligado a distintos elementos que interactúan simultáneamente, aspecto que contribuyó a llamar la atención sobre el inconveniente de reducir el proceso de aprendizaje a rubros específicos, fijos o unidireccionales, por cuanto este proceder minimiza los distintos aspectos que conforman la realidad de las personas. En este mismo periodo, la Física cuántica comienza a formalizar sus ideas y teorías acerca de la constitución de la materia. Esta es sin duda una especie de coincidencia afortunada, por cuanto ambas propuestas contribuyeron a replantear muchos aspectos de la cosmovisión que les precedía, y se constituyeron en mecanismos para formar una idea de que: "la realidad no es lo que parece" (Rovelli 2015), lo cual debe llevarnos a mirar el mundo de una nueva manera (Bohm, 2002).

La Física cuántica, propone que los elementos fundamentales de la materia, denominados cuantos o quarks, se caracterizan por su granularidad, indeterminismo y relacionalidad; lo que remite a lo finito, lo probabilístico y multi-relacional, aspecto que evidencian la dinámica de las partículas (Rovelli, 2015). Según Rovelli, la materia en sus dimensiones microscópicas, se puede representar mediante una "spinfoam" o "espuma de spin", que nos permite considerar que la materia, a nivel de sus unidades más elementales se puede representar como si fueran burbujas o pompas de jabón, que actúan entre sí generando una espuma.

Así, utilizando un modelo similar al propuesto por Rovelli, se propone considerar que una "unidad de aprendizaje", es decir un conocimiento/habilidad, se puede representar como si fuera una "pompa de jabón" y el crecimiento del aprendizaje como una "espuma de pompas de jabón". Cada aprendizaje, que en adelante, se denominará "nodo" y conlleva al concepto de granularidad; donde la cantidad de aprendizajes o nodos que se pueden "adquirir" (o construir) es finito, pero incontable. De esta manera cada aprendizaje se estaría comportando como un quark lo cual es plausible si se considera que: "la cuestión central para la comprensión está en la capacidad de crear modelos de trabajo -elaborados tanto a partir de la percepción como del discurso- con los cuales es posible explicar situaciones o predecir el comportamiento de sistemas (Johnson-Laird, 1983, citado por Greca y Herscovitz, 2002).

Tópicos Educacionais, Recife, v. 25, n.1, p. 103-118, jan/jun. 2019. Disponível em: < https://periodicos.ufpe.br/revistas/topicoseducacionais/> 
Por su parte, Fritjof Capra, en su libro "El Punto Crucial” publicado en 1992, destacó con respecto a la constitución de la materia, que los componentes a nivel micro de la misma, no actúan como entes aislados sino como entes que establecen relaciones entre ellos, de hecho resalta las relaciones en vez de las entidades aisladas, aspecto que posibilita una visión holística de la materia. Estos dos planteamientos, constituyen las premisas que sirven de base para presentar la siguiente metáfora para describir al aprendizaje, basada en la interacción dinámica de todos los factores que intervienen en su proceso, los cuales actuarían de manera análoga a cómo actúa la materia a nivel cuántico.

Se parte de considerar que el aprendizaje consiste en un proceso y no un fin en sí mismo, que implica un "aprender a aprender" y "aprehender", aspectos que deben actuar como el eje central del acto educativo. Este aprender a aprender y aprehender permite al aprendiz establecer las múltiples relaciones entre qué, por qué, cómo, cuándo, dónde, mediante qué recursos, se planteará la "aprehensión" del conocimiento/habilidad en cuestión, basados en la mecánica cuántica yq que esta posibilita el considerar que:

a. El aprendizaje como tal es limitado, es decir finito, solo se puede aprender una cantidad limitada de contenidos/habilidades aunque siempre es posible aprender más de un conocimiento/habilidad específico. De esta manera, el aprendizaje no es continuo, sino es discreto y granular, solo es posible aprender una cantidad limitada de cosas aunque éstas se pueden articular de manera incontable, sin embargo esto no significa que el número de articulaciones sea infinita.

b. El aprendizaje adquirido de un conocimiento/habilidad se manifiesta de forma desplegada, recurrente, estable y separable (Bohm, 2002) pero de manera indeterminada y su uso es probabilístico. No se puede afirmar, a priori, cuándo y de qué manera, el individuo utilizará el conocimiento/habilidad adquirido de forma específica y unidireccional (es decir, determinada), de hecho su manifestación puede darse de forma repentina e influenciado por estímulos que podrían no ser considerados.

c. El aprendizaje alcanzado es un reflejo de la interacción que se da entre los conocimientos/habilidades previos y el proceso de acomodación/asimilación de los nuevos. Este reflejo evidencia que se ha logrado algún nivel de aprehensión 
mental del objeto a ser aprendido. De esta manera, manifestar un conocimiento/habilidad adquirido no es más que un reflejo de que el mismo se encuentra incorporado a las estructuras mentales con que cuenta el individuo.

Por lo anterior, se puede afirmar que solo hay aprendizaje si hay interacciones mentales, pero no se puede garantizar, con certeza absoluta, cuando esto puede ocurrir o manifestarse de manera desplegada, por ello el recibir información no implica que se llegue a generar un conocimiento/habilidad, porque no necesariamente conlleva la interacción entre el objeto de conocimiento/habilidad que se pretende generar con la nueva información aportada y los conocimientos/habilidades existentes en las estructuras mentales del individuo

d. El aprendizaje es lo que es en relación a la capacidad de interacción (uso) que le dé el individuo (relacionalidad). Un mismo aprendizaje puede ser utilizado de maneras distintas en distintas circunstancias, o por distintos sujetos que asumen poseen el mismo conocimiento/habilidad asimilado, es decir, un aprendizaje específico puede ser utilizado en distintos sistemas de relaciones, lo cual conlleva un sesgo de creatividad e incertidumbre que refleja la individualidad de cada persona.

estos aspectos evidencian que el aprendizaje es dinámico, personal, contextual, discreto, creciente, flexible, creativo e innovador; se construye en razón de como el sujeto ponga en marcha sus conocimientos previos en procura de responder a los estímulos (información) que recibe de su entorno, natural, social o interno.

En la construcción de un aprendizaje intervienen las leyes de la dialéctica de manera subyacente e implícita, por cuanto la acumulación cuantitativa de aprendizajes posibilita el dar saltos de calidad que potencian una re-estructuración del aprendizaje acumulado; por cuanto, cada aprendizaje conlleva a una modificación de las estructuras mentales del individuo a modo de síntesis, sin embargo mientras tal síntesis no se logre, el individuo mantiene sus aprendizajes por separado, siendo todos válidos, de manera similar como la Física moderna acepta que dos teorías diferentes pueden ser válidas simultáneamente, de esta manera el individuo enfrenta una unidad y lucha de contrarios, 
entre el "viejo" aprendizaje y el "nuevo", por lo que la construcción/síntesis de un nuevo aprendizaje deviene en la negación de un aprendizaje anterior, lo que permite establecer que la negación de lo negado genera un nuevo aprendizaje.

Por su parte, cualquier nodo se relaciona con otros nodos (tal y como se relacionan las “pompas de jabón”), de manera dinámica, indeterminada y probabilística, relación que se establece a través de las "paredes" de la pompa. Estas paredes se corresponden, a al menos, a los siguientes aspectos: a) cognición, b) emoción/afectividad, c) tiempo/espacio, d) comunicabilidad, e) características fisiológicas, f) estilo de aprendizaje. No se quiere decir con esto que únicamente esos aspectos definen al aprendizaje, sino más bien, que el aprendizaje es el resultado de la combinación dinámica, en una interacción vinculante, simultánea y recíprocamente dependiente, de al menos esos aspectos. Estas "paredes”, al igual que en las pompas reales, son de tamaño irregular, lo que significa que el impacto e importancia que en la construcción de aprendizaje tienen cada uno de los elementos citados, es variable.

De esta manera se pretende superar el dilema de la separación arbitraria y maniqueísta, propios de la modernidad occidental modelada por el pensamiento cartesiano-newtoniano (Bohm, 2002), por cuanto ninguno de los componentes del aprendizaje (o paredes de la pompa) puede ser considerado como más importante que otro, de hecho, todas las paredes de la pompa son necesarias para que esta existe (es decir para que exista el aprendizaje), si una falta, la pompa no se conforma, o lo hace de manera deficiente, y, por lo tanto, no se daría el aprendizaje esperado. Esto no quiere decir que todas las paredes ejerzan la misma influencia para un aprendizaje específico sino que todas son necesarias, pero ejercen un papel diferente donde, a falta de considerar una de las paredes de la pompa, el aprendizaje como tal no se constituye.

Lo anterior reafirma la importancia de comprender, analizar e investigar sobre el papel que juega cada pared de la pompa, pero advierte que no se debe sobreestimar o minimizar ninguna. Por ejemplo, cuando se valora una pared, como la cognitiva, por encima de los demás, se pierde de vista que a pesar de que el aprendiz puede poner en juego todos los componentes que conlleva la cognición, nadie aprende lo que no quiere aprender, aspecto que está ligado a la pared emocional, por eso, dentro del proceso de aprendizaje no se debe asumir de manera marginal el aspecto emocional.

Tópicos Educacionais, Recife, v. 25, n.1, p. 103-118, jan/jun. 2019. Disponível em: < https://periodicos.ufpe.br/revistas/topicoseducacionais/> 
Lo mismo sucede cuando el lenguaje (que como recurso de comunicación, abarca distintos mecanismos que incluyen lo visual, auditivo, táctil, movimiento, etc., y se manifiesta a través de un orden específico) deja de ser considerado como parte inherente del proceso del aprendizaje, incluso Bohm (2002, pág. 60) afirma que: "no sería adecuado aislar el lenguaje como un campo particular de la investigación ni considerarlo como algo relativamente estático, que solo cambia lentamente (o nada en absoluto) cuando uno entra en él”. El lenguaje, cuyo uso es contextual, denota la re-levancia o irre-levancia de los objetos que maneja el aprendizaje (Bohm, 2002) y facilita o dificulta la construcción del conocimiento/habilidad así como su comprensión, en razón del orden con que presenta los conocimientos o habilidades a aprender por cuanto el lenguaje permite la comunicabilidad desde lo intangible, donde las cosas que pensamos mantienen una "cosidad" que permite que sean comunicadas.

Por su parte, al considerar la pared que plantea "el estilo de aprendizaje", cabe destacar que no existe mucha claridad sobre el papel que este juega. Sin embargo se presupone que el estilo de aprendizaje refleja las características individuales referentes al modo preferente o dominante, que manifiesta un individuo cuando aprende (De Lièvre, Temperman, Cambier, Decamps y Depover, 2009; De la Barrera, Donolo, Rinaudo, 2010) pero que no es el único que posee, de ahí que Rieben (2000) considere al estilo de aprendizaje como la preferencia que manifiestan los individuos cuando recurren a uno $\mathrm{u}$ otro proceso para resolver problemas o actuar sobre el entorno. Por su parte Popescu (2008) agrega que, no necesariamente reflejan todas las capacidades cognitivas e intelectuales del individuo, y por su parte, Grasha (2002) considera que el estilo de aprendizaje refleja las disposiciones personales que influyen o influencian en la habilidad del estudiante para tener acceso a la información. A pesar de esta diversidad de criterios, es un hecho que cada individuo aprende de una manera particular.

Los aspectos relativos a la pared espacio/tiempo plantean que el aprendizaje se construye en razón de un tiempo o momento, y en un espacio o lugar específico y particular, pero estos aspectos no necesariamente se manifiestan de manera específica, es decir, el aprendizaje no está condicionado a darse en un momento y lugar precisos (como se espera suceda en el modelo de enseñanza-aprendizaje), por cuanto depende de cuándo y dónde el individuo logre el "insight", es decir, que logre establecer interrelaciones entre 
los elementos nuevos y los viejos que hay en su estructura mental, los cuales pueden ser totalmente asincrónicos con respecto a cuándo y dónde el aprendiz recibió los estímulos.

La identificación de los elementos (paredes) apuntados responde a consideraciones metodológicas, no epistemológicas, y contribuye a justificar por qué sería un error apreciar el aprendizaje dando una preferencia especial a cualquiera de las paredes consideradas por separdo de las demás, ya que tal separación conlleva la posibilidad de cometer errores de interpretación producto de la fragmentación, superficialidad, alcance limitado o irrelevancia, en el análisis realizado, lo cual puede dificultar y hasta impedir el comprender plenamente como se da el aprendizaje, aspectos que no resuelven mediante un acercamiento interdisciplinario, o incluso transdisciplinario, si tales acercamientos conservan a su vez los problemas de fragmentación que implican las diferentes disciplinas (Bohm, 2002). De esta manera se está abogando por una conceptualización holística sobre el aprendizaje.

El aprendizaje debe devenir en el resultado de una apreciación global de un contenido/habilidad, producto de una visión de conjunto (holística) y no como el resultado de una integración por yuxtaposición. El aprendizaje emerge a partir de un análisis e interpretación simultáneo de los diferentes elementos descritos, de ahí la necesidad de tomar en cuenta las diferentes "paredes" de la pompa, por cuanto todas participan en su construcción, re-levancia y significatividad de un aprendizaje. Por lo anterior, el privilegiar una de las "paredes" con respecto a las demás y valorar al aprendizaje como una manifestación desplegada, recurrente, estable y separable desde una sola de las paredes es inconveniente

Adicionalmente, cabe considerar que el aprendizaje está relacionado con el pensamiento en virtud de que el mismo es el reflejo de lo que acontece en la mente del individuo, y que el pensamiento:

es en esencia la respuesta activa de la memoria en cada fase de la vida. Incluimos en el pensamiento las respuestas intelectual, emocional, sensitiva, muscular y física de la memoria. Estos son todos los aspectos de un solo proceso indisoluble. Tratarlos separadamente produce fragmentación y confusión (Bohm, 2002).

De esta manera, la metáfora propuesta permite considerar que las distintas paredes de la "pompa de jabón", que constituyen el aprendizaje, no se manifiestan en las mismas dimensiones o probabilidades, son asimétricas y actúan de manera tanto sincrónica como 
asincrónica (bajo el principio de incertidumbre), lo que hace que el aprendizaje no sea el resultado de una integración forzada o como resultado o una sucesión de eventos, sino un todo, producto del movimiento simultáneo de todas las paredes de la pompa de jabón.

Así, como la estructura de la materia no es mecánica (Rovelli, 2015), la construcción del aprendizaje tampoco lo es, de hecho, en virtud de las actitudes, vínculos y relaciones que el aprendiz establece con el objeto de aprendizaje, es el quien provoca las propiedades y características de tal objeto, lo que implica para el aprendiz un proceso de auto-organización mental de los contenidos/habilidades aprehendidos, propios de la auto-organización humana (Capra, 1992), donde la enseñanza solo constituye una ambientación o climatización para facilitar tal proceso.

A la perspectiva anterior, se debe agregar la consideración de que todo objeto de conocimiento/habilidad puede ser interpretado como un "texto". Para este "texto" el individuo parte de un pre-texto, es decir, parte de la condición de no poseer el texto y de valorar la necesidad o el interés por adquirirlo y esto lo hace en un con-texto específico entendido como las condiciones específicas que permiten el aprehender tal conocimiento/habilidad. Esto hace posible pensar el aprendizaje a partir de un enfoque integral, haciendo hincapié en los principios básicos de la organización y auto organización que conlleva; es decir posibilita valorar al aprendizaje como un proceso de reorganización dinámica de los conocimientos/habilidades que posee el individuo en su esfuerzo por acomodar/asimilar en sus estructuras mentales los nuevos conocimientos/habilidades que enfrenta, sobre los que media (implican para el individuo) interés o necesidad, lo que conlleva una retroalimentación permanente y con ello un crecimiento constante.

Así, la producción de nuevos aprendizajes depende tanto de las características y contenidos del objeto de aprendizaje como del sujeto, lo cual justifica que sea totalmente individual, por lo que, no existen dos aprendizajes iguales y no existen dos personas que puedan aprender de manera exactamente igual, a pesar de las regularidades que puedan presentar tanto los contenidos del aprendizaje como los individuos, es decir, cada quien tiene su pre-texto, texto y con-texto.

La metáfora planteada posibilita además considerar que la construcción del aprendizaje es posible si se le considera como una transacción que resulta de: "la interacción simultánea y recíprocamente dependiente entre componentes múltiples"

Tópicos Educacionais, Recife, v. 25, n.1, p. 103-118, jan/jun. 2019. Disponível em: < https://periodicos.ufpe.br/revistas/topicoseducacionais/> 
(Capra, 1992, pág. 144), transacción en la que participan el individuo y el entorno social y natural en el que vive. Tal transacción debe ser manejada de manera flexible tanto por el aprendiz como por los distintos actores que forman parte de los entornos natural y social, por cuanto los aprendizajes a adquirir, o desarrollar, deben contribuir a dinamizar la comprensión y el quehacer del individuo en el marco de su interacción con el entorno, y en caso del entorno social esto solo es posible si se dan consensos.

Como la construcción de un aprendizaje es probabilístico y depende de las condiciones y características concretas del entorno natural/social en que se desenvuelve el individuo; el mismo se facilita si el aprendiz toma conciencia del carácter multivariable que posee, así como de las relaciones que establece con el entorno natural/social del cual es parte y, de las motivaciones (intereses o necesidades) que direccionan sus acciones. Además, por su naturaleza multirelacional, el aprendizaje no puede ser producto de tan solo una interacción entre los nodos contenidos de un par de burbujas según la metáfora, sino más bien el resultado de la interacción de múltiples nodos, muchas veces mediante aproximaciones sucesivas entre el acervo del individuo (los nodos existentes) y los nuevos elementos que enfrenta, donde cada aproximación aporta y contribuye con una parte de la construcción del conocimiento/habilidad a ser aprendida.

Aunado a lo anterior, debe tomarse en cuenta que el aprendizaje no siempre depende de elementos externos a los que es expuesto el individuo, el mismo puede ser el resultado de una re-lectura de la información, conocimientos o habilidades con que cuenta y que es parte de sus estructuras mentales. Esto significa que el aprendizaje puede ser totalmente asincrónico y manifestarse mucho tiempo después de que el individuo haya recibido la información que se pretende se convierta en un conocimiento o habilidad, incluso aquí vale volver a la Física cuántica como elemento referencial, por cuanto, como lo plantea Rovelli (2015) la Física mucha veces avanza aun cuando no disponga de nuevos datos, ya que el avance puede estar relacionado con la re-lectura de aquellos con los que cuenta. Así basta retomar los conocimientos/habilidades con que se cuenta y re-leerlos de una manera nueva y diferente, dentro de la nube de probabilidades, para que surjan nuevos aprendizajes.

Tópicos Educacionais, Recife, v. 25, n.1, p. 103-118, jan/jun. 2019. Disponível em: < https://periodicos.ufpe.br/revistas/topicoseducacionais/> 
Esto reafirma que los contenidos/habilidades aprendidos pueden re-organizarse y auto-organizarse de manera constante (permanente), lo que lleva a una auto-producción de nuevos conocimientos/habilidades merced a la retroalimentación que tales aspectos conllevan (Musa, 2010) aun cuando no haya nueva información. Esta reorganización evidencia por su parte la importancia que tiene para la construcción de nuevos aprendizajes el que las paredes de cada burbuja puedan modificarse, y por ende potenciar la construcción de un conocimiento/habilidad nueva y diferente.

Se puede afirmar entonces que el aprendizaje no es un cambio "per se" sino relativo, influido por la interacción sujeto/objeto de conocimiento/habilidad; por la relación que tiene el sujeto con el entorno (natural y social e interno), por la relectura que el sujeto haga de sus conocimientos/habilidades, o por la modificación dinámica de cualesquiera de las condiciones (paredes de la pompa) que garantizan su existencia. A partir de lo anterior y como consecuencia del mismo, el aprendizaje implica la transformación del sujeto y del objeto de aprendizaje (conocimiento/habilidad) de manera simultánea; el sujeto adquiere un "bien" que articulado con sus conocimientos, conductas y habilidades previas, lo transforman al cambiar su red neuronal, lo que cambia su visión del mundo y su relación con el mismo, así como su capacidad de comunicabilidad, de percibir, interpretar, y manipular más conocimientos y habilidades; por su parte el objeto de conocimiento/habilidad se transforma al pasar de ser externo al sujeto a ser parte de su acervo conceptual e ingresa en al ámbito de la "cosidad" que existe en la mente del mismo.

Sin lugar a dudas el aprendizaje está ligado a la capacidad del individuo de recibir información (por cualquier vía, intencionada o no, externa o interna), de manera que si la información puede ser relacionada con los conocimientos/habilidades pre-existentes, el procesamiento de dicha información transforma el bagaje acumulado por el individuo y se transforma a sí misma al incorporarse a dicho bagaje, no como algo nuevo, sino como algo diferente. Esto hace que el aprendizaje sea una "cosa en relación a", o "en relación con"; por lo que plantear que el aprendizaje simplemente implica que ahora se conoce o hace lo que antes no se conocía o se hacía, es limitado, fragmentario y responde a un paradigma conductista.

Tópicos Educacionais, Recife, v. 25, n.1, p. 103-118, jan/jun. 2019. Disponível em: < https://periodicos.ufpe.br/revistas/topicoseducacionais/> 
Así, una re-lectura de la definición de aprendizaje, propuesta por Cotton deja claro por qué no es conveniente que dicha definición incluya el término "perdurable", por cuanto esta condición no existe en términos reales, ya que todo aprendizaje es relativo, contextual, dinámico, está en permanente transformación, depende del espacio/tiempo en que que se utiliza, se manifiesta de manera específica, es probabilístico, susceptible de cambiar (ya sea mejorando en su manejo o por las relaciones que conlleva, o perdiéndose por desuso). Esto aclara por qué lo que era importante de aprender hace 100 años no necesariamente lo es ahora, y lo que se aprende ahora podría no ser significativo dentro de 100 años, por cuanto el aprendizaje está ligado a la modificación/transformación del individuo y de su entorno, lo cual modifica las condiciones que llevaron a su construcción, generándose así una espiral creciente, con modificaciones constantes que revelan el carácter dialéctico, dinámico y creciente del mismo

Ahora bien, el aprendizaje como construcción individual en medio de un entorno natural/social/interno, solo tiene sentido si es comunicable, es decir, solo existe en el marco de la interacción con el aprendizaje de otros, o del mismo sujeto en la medida que evidencie la diferencia entre un antes y un después (un pre-texto y el pos-texto). Dado que el aprendizaje obedece, o bien a la necesidad o al interés (aspectos definidos por el sujeto), el mismo solo se puede evidenciar de manera relacional, por lo que no se puede saber realmente que tanto ha aprendido el individuo, es decir, que tanto ha crecido su "espuma de burbujas" hasta tanto no ponga ésta en relación con otros individuos, lo cual evidencia otra conexión con la física cuántica, ya que un sistema físico se manifiesta solo cuando interactúa con otro (Rovelli, 2015), así, un aprendizaje logrado, solo se puede manifestar cuando interactúa con otro aprendizaje. Es decir, un individuo solo puede comprobar ante los demás, o ante sí mismo, que ha adquirido un aprendizaje en la medida que este pueda ser utilizado en razón de su interacción con otros aprendizajes.

Al igual que en la mecánica de las partículas, de las cuales según la Física cuántica, solo podemos ver el trazo que deja la interacción entre ellas, el proceso de aprendizaje de un contenido/habilidad, como tal, no es posible apreciarlo y solo podemos apreciarlo en la interacción con otros aprendizajes. Incluso un individuo no puede detallar con precisión absoluta como se dio, por lo que lo único posible visualizar es el uso que el individuo hace de dicho contenido/habilidad con respecto a otros contenidos/habilidades, es decir, no es posible observar o detectar como se efectuó el aprendizaje, con que 
conocimientos/habilidades previos fue asociado el nuevo elemento (la expectativa del enseñante es que lo asocie con los conocimientos/habilidades previos que asume debe tener el aprendiz), por ello solo se puede seguir el rastro de este proceso en la medida que el aprendiz exteriorice (comunique) lo que considera que hizo para "conectar" el contenido nuevo con los contenidos previos que posee.

Por último, cabe destacar que, el hecho que desde una perspectiva biológica el envejecimiento fisiológico constituye un proceso que ocurre a lo largo de la vida la vida y no únicamente como expresión de la última etapa de la edad adulta, el cual abarca el conjunto de cambios biológicos que favorecen pérdidas funcionales (Hayflick, 1994, citado por Libro Blanco del envejecimiento activo, 2011), evidencia que el aprendizaje enfrenta problemas para su crecimiento, por cuanto el deterioro da la pared fisiológico/biológico afecta otras paredes como la cognitiva y la emocional, y permite reafirmar que el aprendizaje no es infinito, es decir, es imposible que el aprendizaje pueda crecer ilimitadamente.

Y es que al considerar la "pared" de la burbuja relativa a lo biológico, conforme el individuo envejece tal pared, en muchos casos tiende a hacerse cada vez más delgada y débil, dificultando las interacciones entre los nodos de aprendizaje, e incluso mostrando tendencia a romperse. Para retrasar este aspecto, y posibilitar al individuo que envejece pueda mantenerse aprendiendo, es imprescindible que el mismo pueda hacerlo de la manera lo más activamente posible, lo cual se logra en la medida que el envejecente haga cosas, participe en actividades culturales, artísticas, físicas, sociales, etc., que mantengan activa su mente, por cuanto: "La actividad intelectual y el aprendizaje no sólo son posible en el proceso de envejecimiento sino recomendables para desarrollar mayor plasticidad cognitiva a lo largo de toda la vida adulta” (Arnay, Marrerro y Fernández, 2013)

\section{A modo de resumen}

La metáfora presentada para describir el aprendizaje con base en la física cuántica, propone que el aprendizaje de un contenido/habilidad específico se comporta como una partícula de material, por lo que no es más que una abstracción que solo se puede definir y observar a través de la interacción que desarrolla con otros aprendizajes, tal y como lo planteó Niels Bohr con respecto a las partículas de materia (Rovelli, 2015). Este enfoque

Tópicos Educacionais, Recife, v. 25, n.1, p. 103-118, jan/jun. 2019. Disponível em: < https://periodicos.ufpe.br/revistas/topicoseducacionais/> 
permite re-conceptualizar el aprendizaje como un todo y no como la suma de sus partes, aspecto recurrente desde la perspectiva cartesiana-newtoniana, (Capra, 2002), y es susceptible de poder analizarse mediante el método dialéctico, por cuanto las tres leyes que definen este método se ajustan al modelo de crecimiento de la "espuma de burbujas", donde cada nuevo aprendizaje, se une a los que previamente posee el individuo, modifica toda la estructura existente y abre espacios a partir de la negación, como lo plantea Bachelard, para nuevas consideraciones y aprendizajes.

La metáfora considera como elementos identificables para el aprendizaje: la cognición, la emoción, la comunicabilidad, las características fisiológicas, los estilos de aprendizaje, el espacio/tiempo; posee un carácter holístico, tal y como lo demanda el nuevo paradigma de la física cuántica; no afirma en modo alguno que sean los únicos, pero sí que todos ellos deben ser tomados en cuenta y que la no consideración de cualquiera de ellos desvirtúa lo que puede entenderse por aprendizaje, por cuanto el mismo no ocurría como tal.

El considerar el aprendizaje desde la perspectiva de la Física cuántica no quiere decir que se deba rechazar la visión cartesiana-newtoniana ni se rechaza la importancia que tiene el estudio e investigación que por separado ejerce cada uno de estos componentes en la construcción del aprendizaje, por cuanto: “el objetivo no es sustituir un modo por el otro, sino más bien pasar del énfasis excesivo puesto en cualquiera de los dos a un mayor equilibrio entre ambos" (Capra, 1992), lo que se persigue con la metáfora es re-dimensionar al aprendizaje como el resultado de las múltiples interacciones y vínculos entre los distintos componentes descritos y no como su suma aritmética. Esto permite construir puentes que facilitan la transición de un nivel u orden a otro superior, y el comprender que el objetivo del proceso educativo debe centrarse en potenciar en el educando su capacidad de aprender a aprender y dejar de lado el modelo centrado en la presentación de contenidos por parte del enseñante.

El estudio especializado de cómo actúa cualquiera de las paredes de la burbuja, producto de las investigaciones sobre el papel de la cognición o de las emociones a modo de ejemplos, deben contribuir a ver al aprendizaje como un todo. El conocimiento detallado de cada pared posibilita una mejor comprensión del comportamiento del todo 
por cuanto es posible visualizar con mucha más claridad el papel que cada pared juega y como se relaciona con las demás, lo que resulta contraproducente es asumir que basta con conocer sobre una o algunas de las paredes y que estas visiones por separado son suficientes para explicar el fenómeno del aprendizaje.

\section{Referencias:}

Arnay, J.; Marrero, J.; Fernández, I. (2013). Educación y envejecimiento: el envejecimiento constructivo. Revista Informació Psicológica. $\mathrm{N}^{\circ} 104$, JulioDiciembre. Recuperado de www.informaciopsicologica.info/OJSmottif/index.php/leonardo/article/view/30

Bohm, D. (2002). La totalidad y el orden implicado. Editorial Kairós, Barcelona, España.

Cantú, I. (2003). El estilo de aprendizaje y su relación con el desempeño académico en los estudiantes de arquitectura de la Universidad de Nuevo León. Revista Internacional de Estudios en Educación. Vol 3, $n^{\circ} 2$.

Capra, F. (1991). El nuevo paradigma ecológico. Nueva Conciencia, $\mathrm{N}^{\circ} 22$. Recuperado de http://absorto.sdf.org/el_nuevo_paradigma_ecologico.html

Capra, F. (1992). El punto crucial. Editorial Troquel. Buenos Aires, Argentina.

De la Barrera, M., Donolo, D., Rinaudo, M. (2010). Estilos de aprendizaje en alumnos universitarios: peculiaridades al momento de aprender. Revista Estilos de Aprendizaje, $n^{\circ}$ 6, Vol 6, octubre de 2010. Universidad Nacional de Educación a Distancia. España. Recuperado de www.uned.es/revistaestilosdeaprendizaje/

De Lièvre, B.; Temperman G.; Cambier, J.; Decamps, S.; Depover, Ch. (2009). Analyse de l'influence des styles d'apprentissagesur les interactions dans les forums collaboratifs. Develotte C., Mangenot F., Nissen E. (2009, coord.) Actes du colloque Epal 2009 (Echanger pour apprendre en ligne: conception, instrumentation, interactions, multimodalité), Université Stendhal - Grenoble 3, 5-7 juin 2009. Recuperado de w3.ugrenoble3.fr/epal/dossier/06_act/pdf/epal2009-delievre-et-al.pdf

García, J. (2011). La incidencia de los estilos de aprendizaje de los estudiantes de ingeniería Eléctrica en la Sede Guanacaste de la Universidad de Costa Rica con el aprendizaje del cálculo diferencial. Tesis para obtener el grado de Maestro en Educación. Escuela de Graduados en Educación. Instituto Tecnológico y de Estudios Superiores de Monterrey. Nuevo León, México.

Tópicos Educacionais, Recife, v. 25, n.1, p. 103-118, jan/jun. 2019. Disponível em: < https://periodicos.ufpe.br/revistas/topicoseducacionais/> 
Grasha, A. (2002). Teaching with style. A practical guide to enhancing learning by understanding teaching and learning Styles.Alliance Publishers. USA. Recuperado de www.ilte.ius.edu/pdf/teaching_with_style.pdf

Greca, I. y Herscovitz, V. (2002). Construyendo significados en mecánica cuántica: fundamentación y resultados de una propuesta innovadora para su introducción en el nivel universitario. Revista Enseñanza de las Ciencias. Instituto de Física, Universidad Federal do Rio Grande do Sul. Recuperado de www.raco.cat/index.php/Ensenanza/article/download/21818/21652

Libro Blanco sobre el envejecimiento activo. (2011). Ministerio de Sanidad, Política Social e Igualdad Secretaría General de Política Social y Consumo. Instituto de Mayores y Servicios Sociales (IMSERSO). Madrid. España

Musa, J. A. (2010). Cognición cuántica y aprendizaje diamantino. Un enfoque holístico del proceso de enseñanza-aprendizaje. Ponencia presentada en el Congreso Iberoamericano de Educación, Metas 2021. Buenos Aires, Argentina. Recuperado como RLE2496_Mussa.pdf de www.adeepra.org.ar/congresos/Congreso\%20IBEROAMERICANO/ACCESO

Popescu, E. (2008). Dynamic adaptive hypermedia systems for e-learning. Doctorat Tis. Université de Craiova, Roumanie. Recuperado de www.tel.archivesouvertes.fr/docs/00/34/34/60/PDF/thesis_ElviraPopescu.pdf

Rieben, L. (2000). À quelles conditions la notion de style d'apprentissagepeutelledevenirheuristique pour le champ de l'éducation?Le style d'apprentissage.Volume XXVIII, $N^{o} 1 . \quad$ Recuperado de www.acelf.ca/c/revue/revuehtml/28-1/08-rieben.html

Rovelli, C. (2015). La realidad no es lo que parece. TusQuets Editores. Barcelona, España.

Saldaña, M. (2010). Estilos de aprendizaje y rendimiento académico en alumnos que cursaron genética clínica en el periodo de primavera 2009 en la Facultad de Medicina de la Benemérita Universidad Autónoma de Puebla. Revista estilos de Aprendizaje $n^{o}$ 5, Vol 1 abril 2010. Recuperado de www.uned.es/revistaestilosdeaprendizaje/numero_5/.../lsr_5_articulo_3.p 